\title{
Investigating a playing strategy for drumming using surface electromyograms
}

\author{
Takuya Fujisawa ${ }^{1, *}$ and Masanobu Miura ${ }^{2, \dagger}$ \\ ${ }^{1}$ Graduate School of Science and Technology, Ryukoku University, Japan \\ ${ }^{2}$ Faculty of Science and Technology, Ryukoku University, Japan
}

(Received 14 December 2009, Accepted for publication 14 February 2010)

Keywords: Drums, Rhythm, Single-stroke, EMG, Playing strategy

PACS number: 43.45.Hi, 43.75.St [doi:10.1250/ast.31.300]

\section{Background}

In drumming, the skill of controlling drumsticks correctly is required to play rhythms without making any mistakes at suitably dynamic levels of sound. The results of the analysis of drummers' movements from visual information, such as motion-capture or camera recordings, have been reported in past studies [1,2]. However, analysis using biological information has neither been reported nor suggested.

\section{Aims}

2.1. Playing strategy

In drumming, it is assumed that considering subsequent performance is essential for a drummer. For example, having a drummer's mental rehearsal of the subsequent beat/measure is an effective way of reducing mistakes. It is additionally assumed that considering the balance of the internal and external power of a player is also essential for the drummer. An example of the player's internal power is the stroking power, and that of the player's external power is the rebound from the drumhead. Taking advantage of external power such as the rebound from the drumhead and weight of the drumstick reduces the strain of the drummer in terms of muscle control. In this study, such considerations are called "playing strategy." Trained drummers are thought to have different playing strategies, such as a short-term playing strategy (e.g., every stroke) and a long-term playing strategy (e.g., every excerpt).

\subsection{Method of analysis of playing strategy}

Playing strategy is based on a drummers' skill and experience, so it is implemented with or without a drummer being conscious of it. By recording electromyograms (EMGs), information can be obtained about the drummer's conscious and unconscious movements, so analyzing the EMGs is thought to be suitable for investigating drummers' playing strategies. In addition, the amount of EMG signals is proportional to the amount of muscle strain. Therefore, by analyzing the EMGs of the arms when playing the drums, it is possible to investigate the way the arms control the drumsticks, in terms of muscle laxity and strain. The purpose of this study is to investigate the relationship between trained drummers' playing strategies and muscle movements by recording their EMGs while playing the drums.

\footnotetext{
*e-mail: uho_auc@yahoo.co.jp

†e-mail: miura@rins.ryukoku.ac.jp
}

\subsection{Summary of investigation}

To investigate the relationship between playing strategies and muscle movements, three different experiments are conducted: an investigation concerning the relationship between drumhead-condition/drumstick-condition and shortterm playing strategy (EXP1), an investigation concerning the relationship between length/weight of the drumstick and short-term playing strategy (EXP2), and an investigation concerning the relationship between continual playing and short-term/long-term playing strategy (EXP3). EXP1 and EXP2 concern short-term playing strategy, and EXP3 concerns about short-term and long-term playing strategies.

3. EXP1: Investigation of relationship between drumhead-condition/drumstick-condition and shortterm playing strategy

3.1. Experiment objective (EXP1)

When playing drums, the drumstick is controlled by not only the player's internal power but also the player's external power, such as rebound from the drumhead, so a player considers the balance of internal and external power while keeping up with changes in the performance condition for every stroke. Therefore, it is assumed that such consideration corresponds to short-term playing strategy. In this experiment, we investigate the relationship between drumhead-condition/ drumstick-condition and short-term playing strategy. Taking advantage of rebound from the drumhead and the weight of the drumstick reduces the strain on the drummer when playing drums. By comparing EMGs under a "normal condition" and a "removing external power condition," it is possible to investigate how the player controls the drumstick under the normal condition.

\subsection{Task and subjects (EXP1)}

Three amateur drummers (D1-D3: more than four years of drumming experience) and three novice drummers (B1-B3: less than one year of drumming experience) participated in EXP1. They were asked to play a single stroke with three different conditions, that is, "Real playing (R.condition)," "Stick-only playing (S.condition)" and "Imitative playing (I.condition)" at three different tempi of 80, 100, and 120 bpm. Finally, 18 trials (two trials for each condition and tempo) were conducted with each player. R.condition is a conventional playing condition in which both the drumhead and drumstick are used. S.condition is a playing condition in which the drumstick is used but not the drumhead. I.condition is a playing condition in which player uses half length of the 


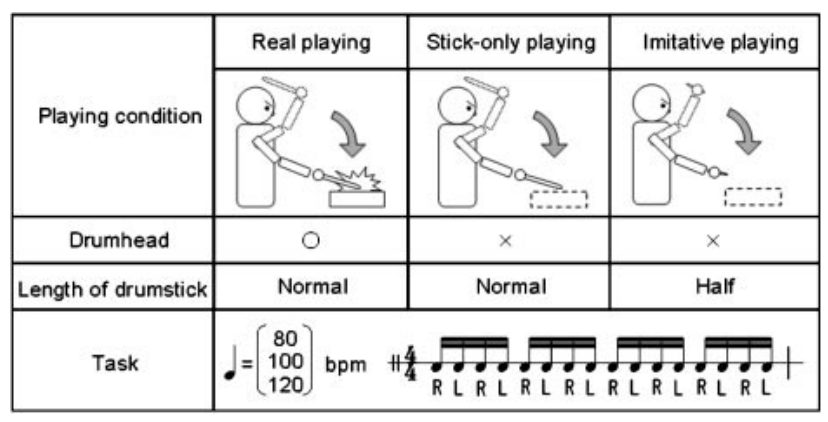

Fig. 1 Task of experiment 1.

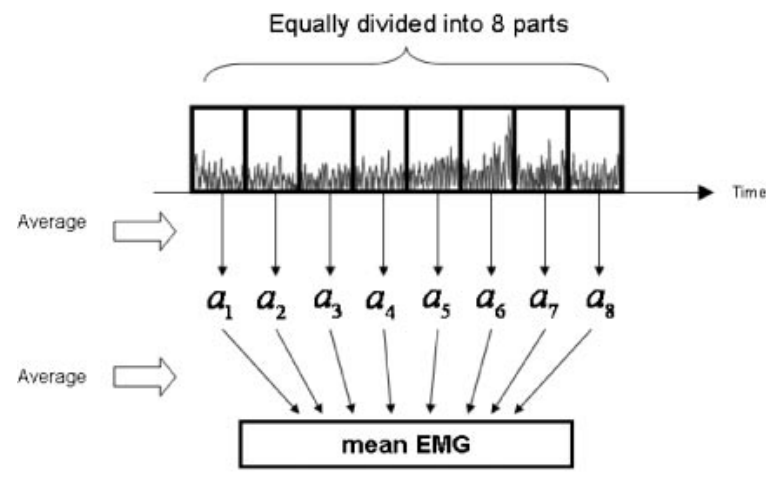

$a_{n}$ : mean EMG of $n$th part

Fig. 2 Method of calculating mean EMG.

drumstick. The flexor carpi ulnaris muscle of both arms, metronome signal, and oscillation of the drumhead were measured. The movement of the flexor carpi ulnaris muscle corresponds to the movement of bending the wrist forward [3].

\subsection{Method of analysis (EXP1)}

In order to analyze the player's movement, the mean EMG in a recording is calculated. The absolute values of the EMG for eight measures are averaged for one recording, and the mean EMG for that recording is calculated. The normalized EMG signal using maximum isometric tension is calculated. The method of calculating the mean EMG is shown in Fig. 2.

3.4. Results (EXP1)

The mean EMG of R.condition was compared with those of S.condition and I.condition. In the same way, the mean EMG of I.condition was compared with that of S.condition. The results of the comparison of mean EMGs are shown in Table 1, where $p$ represents the probability value.

Table 1 confirms that all players' mean EMGs in the case of R.condition were significantly less than those of S.condition. It also confirmed that all trained drummers' and one novice drummer's mean EMGs in the case of R.condition were significantly less than those of I.condition.

\subsection{Discussion (EXP1)}

As a result of EXP1, in the case of all players, it is confirmed that mean EMGs in the case of R.condition were significantly less than those of S.condition. S.condition is a playing condition in which the player does not use the
Table 1 Results of comparison of mean EMG (EXP.1).

\begin{tabular}{cccc}
\hline & $\mathrm{R}<\mathrm{S}$ & $\mathrm{R}<\mathrm{I}$ & $\mathrm{I}<\mathrm{S}$ \\
\hline $\mathrm{D} 1$ & $* *$ & $* *$ & $* *$ \\
$\mathrm{D} 2$ & $* *$ & $* *$ & - \\
$\mathrm{D} 3$ & $* *$ & $* *$ & - \\
$\mathrm{B} 1$ & $* *$ & $* *$ & $* *$ \\
$\mathrm{~B} 2$ & $* *$ & - & $* *$ \\
$\mathrm{~B} 3$ & $* *$ & - & $* *$ \\
\hline
\end{tabular}

$(* *: p<0.01,-$ not significant)

drumhead, so the player cannot take advantage of the rebound from the drumhead. In playing under S.condition, players are required to stop the drumsticks near an imaginary drumhead. Hence, the amount of EMG in S.condition should be higher than that in R.condition. Therefore, the drummers take advantage of the rebound from the drumhead for drumstick control.

In the case of all trained drummers, it is confirmed that the mean EMG in the case of R.condition was significantly less than that of I.condition. In playing under I.condition, half the length of the drumstick is used, so more of the player's internal power is required to lift their hands. Thus the EMG in I.condition should be higher than that in R.condition. Therefore, the trained drummers may possibly take advantage of the weight and length of drumsticks for drumstick control.

\section{EXP2: Investigation of relationship between length/} weight of drumstick and short-term playing strategy

\subsection{Experiment objective (EXP2)}

In EXP1, it was confirmed that the mean EMG in the case of R.condition was significantly less than that of I.condition. The results of EXP1 showed that trained drummers may take advantage of the weight or/and length of drumsticks for drumstick control, but it is not clear whether the results of EXP1 are based on the difference in drumstick length or the difference in drumstick weight. This experiment is conducted to investigate the relationship between the length/weight of the drumstick and EMGs.

\subsection{Task and subjects (EXP2)}

Three amateur drummers (D4-D6) and three non-drummers (ND1-ND3) participated in EXP2. They were asked to play a single stroke with under different conditions, that is, "Normal-length, Normal-weight (NN)," "Short-length, Normal-weight (SN)," "Normal-length, Light-weight (NL)," "Short-length, Light-weight (SL)," "Normal-length, Heavyweight (NH)," and "Short-length, Heavy-weight (SH)" at the three tempi of 80, 100, and $120 \mathrm{bpm}$. Finally, 36 trials (two trials for each condition and tempo) were conducted with each player. The task of EXP2 is shown in Fig. 3.

4.3. Analysis and results (EXP2)

In order to analyze player's movements, the mean EMG in a recording is calculated. The mean EMG is calculated in the same way as in EXP1.

The multiple regression analysis is conducted to investigate how the length and weight of the drumstick influence the player's EMG. In the multiple regression analysis, a variable based on the length of the drumstick (LOD) and 


\begin{tabular}{|c|c|c|c|c|c|c|}
\hline Playing condition & NN & SN & NL & SL & $\mathrm{NH}$ & SH \\
\hline Length & Normal & Half & Normal & Half & Normal & Half \\
\hline Weight & $44[g]$ & $26[g]$ & $25[g]$ & 16 [g] & 110 [g] & $60[\mathrm{~g}]$ \\
\hline Task & & bpm & & & & \\
\hline
\end{tabular}

\begin{tabular}{|ll|}
\hline $\mathrm{NN}=$ Normal-length, Normal-weight & $\mathrm{SN}=$ Short-length, Normal-weight \\
$\mathrm{NL}=$ Normal-length, Light-weight & $\mathrm{SL}=$ Short-length, Light-weight \\
$\mathrm{NH}=$ Normal-length, Heavy-weight & $\mathrm{SH}=$ Short-length, Heavy-weight \\
\hline
\end{tabular}

Fig. 3 Task of experiment 2.

another variable based on the weight of the drumstick (WOD) were introduced into the regression equation to estimate the mean EMG for each group of players (drummers and nondrummers).

In the case of drummers, the result of the multiple regression analysis indicated that the two variables were significantly related to the mean EMG $\left(R_{\mathrm{D}}=0.765\right.$, $R_{\mathrm{D}}^{2}=0.530$, LOD $: \beta_{\mathrm{LOD}, \mathrm{D}}=-0.613[p<0.01]$, WOD : $\left.\beta_{\mathrm{WOD}, \mathrm{D}}=0.765 \quad[p<0.01]\right)$. Specifically, the regression coefficient of LOD was a negative value. On the other hand, in the case of non-drummers, the result of multiple regression analysis indicated that only WOD was significantly related to the mean EMG $\left(R_{\mathrm{ND}}=0.802, \quad R_{\mathrm{ND}}{ }^{2}=0.595, \quad\right.$ LOD : $\beta_{\mathrm{LOD}, \mathrm{ND}}=-0.276 \quad$ [not significant], WOD $: \beta_{\mathrm{WOD}, \mathrm{ND}}=$ $0.761[p<0.01])$.

\subsection{Discussion (EXP2)}

It is confirmed that LOD and WOD are significantly related to the mean EMG, and the regression coefficient of LOD was negative only for trained drummers. This indicates that only the mean EMG of trained drummers is in inverse proportion to the length of the drumstick and directly proportional to the weight of drumstick. Therefore, considering the result of EXP1, trained drummers may possibly take advantage of the length of the drumstick for drumstick control.

\section{EXP3: Investigation of relationship between continual playing and short-term/long-term playing strategy}

\subsection{Experiment objective (EXP3)}

When playing drums, trained drummers are assumed to consider their muscle endurance having time and space on their side throughout a piece of music. Therefore, it is assumed that such consideration corresponds to long-term playing strategy. In this experiment, we investigate the relationship between continual playing and long-term playing strategy. In addition, data obtained from this experiment can be applied to investigate short-term playing strategy, hence we also investigate the relationship between continual playing and short-term/long-term playing strategy.

5.2. Task and subjects (EXP3)

Three trained drummers (D7-D9) and three non-drummers (ND4-ND6) participated in an experiment to record their surface EMGs while playing single strokes at three different tempi of 80,100 , and $120 \mathrm{bpm}$. Finally, 6 trials (two trials for each tempo) were conducted for each player.

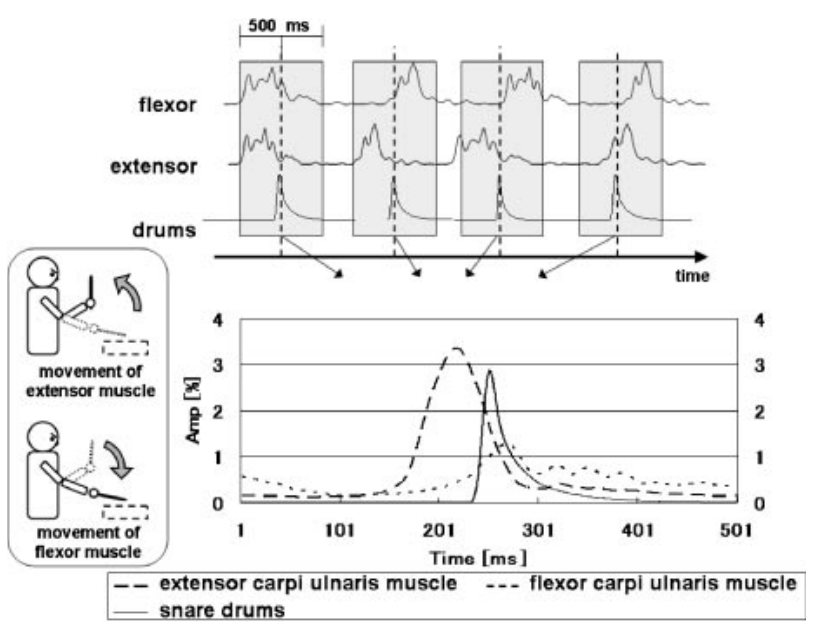

Fig. 4 Method of calculating one-stroke mean EMG.

All the participants were right-handed. They were asked to play four beats for three minutes at the tempi denoted above. The flexor carpi ulnaris muscle of both arms, extensor carpi ulnaris muscle of both arms, metronome signal, and oscillation of the drumhead were all measured. The movement of the flexor carpi ulnaris muscle corresponds to the movement of bending the wrist forward, and the movement of extensor carpi ulnaris muscle corresponds to the movement of bending the wrist backward.

5.3. Analysis and results (EXP3)

5.3.1. Short-term playing strategy

To analyze the short-term playing strategy, the mean EMG in the range of around $500 \mathrm{~ms}$ centered at the time the drumstick strikes the drum is called "one-stroke mean EMG" and is compared for each measured muscle and each drummer. One-stroke mean EMG is the average of waveform for all strokes through one trial. By analyzing one-stroke mean EMG, we can investigate the players' muscle control in playing a stroke. The method of calculating one-stroke mean EMG is shown in Fig. 4.

Table 2 shows the results of a comparison between onestroke mean EMGs of the flexor carpi ulnaris muscles and those of extensor carpi ulnaris muscles for all participants, where $p$ represents the probability value. Table 2 also shows the arm movement in terms of muscle for each participant. The data in Table 2 confirms that significant differences in the one-stroke mean EMG of all participants' extensor carpi ulnaris muscles are less/more than those of flexor carpi ulnaris muscles. "Flexor dominance" is indicated by the one-stroke mean EMG of the extensor carpi ulnaris muscle being less than that of the flexor carpi ulnaris muscle. On the other hand, "Extensor dominance" is indicated by the one-stroke mean EMG of the flexor carpi ulnaris muscle being less than that of the extensor carpi ulnaris muscle.

5.3.2. Long-term playing strategy

To investigate the long-term playing strategy, mean EMGs during the first and the last one minute are compared. Table 3 shows the results of a comparison between mean EMGs during the first and the last one minute. 
Table 2 Result of comparison between one-stroke mean EMG of flexor muscle and one-stroke mean EMG of extensor muscle.

\begin{tabular}{|c|c|c|}
\hline & Left arm & Right arm \\
\hline D7 & $\begin{array}{c}\text { Flexor dominance } \\
(\text { Flexor }>\text { Extensor } * *)\end{array}$ & $\begin{array}{c}\text { Flexor dominance } \\
(\text { Flexor }>\text { Extensor } * *)\end{array}$ \\
\hline D8 & $\begin{array}{c}\text { Flexor dominance } \\
(\text { Flexor }>\text { Extensor } * *)\end{array}$ & $\begin{array}{c}\text { Flexor dominance } \\
(\text { Flexor }>\text { Extensor } * *)\end{array}$ \\
\hline D9 & $\begin{array}{c}\text { Extensor dominance } \\
(\text { Flexor }<\text { Extensor } * *)\end{array}$ & $\begin{array}{c}\text { Extensor dominance } \\
(\text { Flexor }<\text { Extensor } * *)\end{array}$ \\
\hline ND4 & $\begin{array}{c}\text { Extensor dominance } \\
(\text { Flexor }<\text { Extensor } * *)\end{array}$ & ( \\
\hline ND5 & $\begin{array}{c}\text { Flexor dominance } \\
(\text { Flexor }>\text { Extensor } * *)\end{array}$ & $\begin{array}{c}\text { Extensor dominance } \\
(\text { Flexor }<\text { Extensor } * *)\end{array}$ \\
\hline ND6 & $\begin{array}{c}\text { Extensor dominance } \\
(\text { Flexor }<\text { Extensor } * *)\end{array}$ & $\begin{array}{c}\text { Extensor dominance } \\
(\text { Flexor }<\text { Extensor } * *)\end{array}$ \\
\hline
\end{tabular}

$(* *: p<0.01,-$ : not significant $)$

\subsection{Discussion (EXP3)}

\subsubsection{Short-term playing strategy}

Table 2 confirms that four players have the same style of motor control in both arms. Specifically, all trained drummers exhibit this feature, whereas only one non-drummer does, indicating that trained drummers use the same style of motor control in both arms when playing the drums. Conforming right arm movements to left arm movements is thought to be essential in playing the drums without making mistakes throughout a piece of music. In short, trained drummers play drums with the same style of motor control in both arms under the short-term playing strategy.

\subsubsection{Long-term playing strategy}

Table 3 confirms that non-drummers' mean EMGs during the last one minute are significantly higher than those of the first one minute. Such results were obtained only for nondrummers. This indicates that trained drummers are sufficiently relaxed when playing the drums throughout a piece of music. That is to say, trained drummers play the drums with less strain in terms of muscle control than non-drummers under the long-term playing strategy.

\section{Conclusion}

Three types of experiments, EXP1, EXP2 and EXP3, were carried out to investigate short-term and long-term playing
Table 3 Result of comparison between mean EMGs during the first and the last one minute.

\begin{tabular}{cc}
\hline & $\begin{array}{c}\text { Number of mean EMG values } \\
\text { with significant difference } \\
\text { (first one minute }<\text { last one minute) }\end{array}$ \\
\hline Drummer & $17 / 36(42 \%)$ \\
Non-drummer & $26 / 36(72 \%)$ \\
\hline
\end{tabular}

strategies. As the features of short-term playing strategy, trained drummers exhibit the same style of motor control in both arms during playing and experience the least strain under R.condition than under S.condition and I.condition. As a feature of long-term playing strategy, trained drummers experience less strain in terms of muscle control during a continual playing performance. A few novices or nondrummers showed a few of these features, whereas all trained drummers showed all of these features. Therefore, it is expected that trained drummers play the drums taking various factors into consideration, such as less strain in terms of muscle control.

Using surface EMG, we were able to investigate drummers' movements in terms of physiological mechanisms. Future work will focus on investigating using not only biological but also visual information.

\section{Acknowledgements}

This study is partly supported by the High-Tech Research Center Project for Private Universities matching fund subsidy from the Japanese Ministry of Education, Culture, Sports, Science and Technology (MEXT) and Research for Promoting Technological Seeds from the Japan Science and Technology Agency (JST).

\section{References}

[1] E. Altenmüller, M. Wiesendanger and J. Kesselring, Music Motor Control and the Brain (Oxford University Press, Oxford, 2006), pp. 125-138.

[2] S. Dahl and E. Altenmüller, "Motor control in drumming: Influence of movement pattern on contact force and sound characteristics," Proc. Acoustics 08, pp. 1489-1494 (2008).

[3] A. O. Perotto, translated by R. Kashimori, Anatomical Guide for the Electromyographer. The Limbs and Trunk (Nishimura Co. Ltd., Niigata, 2003) (in Japanese). 